\title{
High Yield Selective Induction of Uterine Endometrial Adenocarcinomas in CD-1 Mice by $\boldsymbol{N}$-Ethyl- $\boldsymbol{N}^{\prime}-$ nitro- $N$ - nitrosoguanidine Combined with $17 \beta$-Estradiol
}

\author{
Seiichi Iizuka ${ }^{1,2}$, Susumu Nishimura ${ }^{2}$, Masakazu Takahashi², Jin Ando $^{2}$, Midori Yoshida ${ }^{2}$, \\ Kayoko Kudoh ${ }^{2}$, Katsuhisa Shiraki ${ }^{2}$, and Akihiko Maekawa ${ }^{2}$ \\ ${ }^{1}$ New Drug Discovery Laboratory, Tsumura \& Co., 3586 Yoshiwara, Ami-machi, Inashiki-gun, Ibaraki 300-1192, Japan \\ ${ }^{2}$ Department of Pathology, Sasaki Institute, 2-2 Kanda-Surugadai, Chiyoda-ku, Tokyo 101-0062, Japan
}

\begin{abstract}
In order to assess a combined carcinogen and hormone protocol for selective induction of uterine adenocarcinomas in mice, 79 illumination-induced persistent estrous CD-1 mice, divided into four groups, were treated with $N$-ethyl- $N^{\prime}$-nitro$N$-nitrosoguanidine (ENNG) and/or $17 \beta$-estradiol $\left(\mathrm{E}_{2}\right)$. Groups 1 and 3 were given a single intra-uterine administration of polyethylene glycol (PEG) at 10 weeks of age, while Groups 2 and 4 received ENNG (12.5 mg/ $\mathrm{kg}$ ), dissolved in PEG. Mice of Groups 3 and 4 were also implanted with $E_{2}$ pellets s.c. one week earlier and the pellets were once renewed after 8 weeks. At 15 weeks after the ENNG treatment, the mice were killed for histopathological and endocrinological examination. All groups demonstrated endometrial proliferative lesions, although no severe hyperplasias or adenocarcinomas were found in the control group (Group 1). The incidences of adenocarcinomas in Groups 2, 3, and 4 were $6(1 / 19), 25(5 / 20)$, and 55\% (11/ 20 ), respectively, those for Groups 3 and 4 being significant as compared to the lack in Group 1. The $E_{2}$ : progesterone $\left(E_{2}\right.$ : $P)$ ratios in Groups 3 and 4 were also significantly increased. These results indicate that an increased $E_{2}: P$ ratio is important for endometrial adenocarcinoma development in CD-1 mice, acting with or without chemical carcinogen initiation to cause uterine cancer development. The relatively short duration, and specifically high yield mean that the present protocol has advantages for two-stage uterine carcinogenicity studies in mice. (J Toxicol Pathol 1999; 12: 177 182)

Key words: Endometrial adenocarcinoma, $N$-Ethyl- $N^{\prime}$-nitro- $N$-nitrosoguanidine (ENNG), 17 $\beta$-Estradiol $\left(\mathrm{E}_{2}\right), 17 \beta-$ Estradiol : progesterone $\left(\mathrm{E}_{2}: \mathrm{P}\right)$ ratio, $\mathrm{CD}-1$ mouse
\end{abstract}

\section{Introduction}

Uterine endometrial adenocarcinoma is one of the most common malignant tumors in women. While details of its etiology remain to be clarified, hormones such as estrogens are considered to be most important $t^{1,2}$. There have been many attempts to establish an animal model for human endometrial adenocarcinomas in rodents treated with various chemical carcinogens and/or hormones (either alone or in combination) ${ }^{3}$. In mice, but not rats, induction of uterine adenocarcinomas by estrogen alone has been demonstrated ${ }^{4}$, although at low yield as with chemical carcinogens in general in rodents.

Previously we succeeded in causing a high incidence of uterine endometrial adenocarcinomas by a single intra-uterine administration of $N$-ethyl- $N^{\prime}$-nitro- $N$-nitrosoguanidine (ENNG) in Donryu rats. ${ }^{5}$ In mice, Niwa et al. ${ }^{6}$ earlier reported a good yield with a carcinogen-initiator/ estrogen-promoter regimen. We also successfully produced endometrial carcinomas in illumination-induced persistent estrous CD-1 mice by a single intra-uterine administration of $N$-ethyl- $N$-nitrosourea (ENU), following subcutaneous implantation of a $17 \beta$-estradiol $\left(\mathrm{E}_{2}\right)$ pellet ${ }^{7}$, but lung adenomas were also found and the question of the influence of estrogen alone was not clarified. It is well known that ENU has a wide target organ spectrum in mice ${ }^{8}$. In order to selectively induce uterine cancers at high incidence and also elucidate the effects of estrogen alone, a similar experiment

Received : 16 July 1999, Accepted : 10 September 1999 was here performed in CD-1 mice using ENNG instead of ENU.

\section{Materials and Methods}

\section{Animals and housing conditions}

Female CD-1 mice, 4 weeks of age, were purchased from Charles River Japan Inc. (Atsugi, Kanagawa). They were housed 5 animals to a plastic cage and kept in an airconditioned animal room at $21 \pm 2^{\circ} \mathrm{C}$ temperature and $55 \pm$ $10 \%$ humidity under a $12 \mathrm{~h} / 12 \mathrm{~h}$ light/dark cycle, and maintained on a basal diet CRF-1 (Oriental Yeast Co., Ltd., Tokyo) and tap water ad libitum before use. At 7 weeks of age, all mice were subjected to $24 \mathrm{~h}$ light exposure and thereafter they were maintained under this condition throughout the experiment. Vaginal smears from all mice were checked every morning from 7 to 9 weeks of age, and a total of 79 animals exhibiting persistent estrus were employed for the experiment, these being divided into 4 groups (Fig. 1).

\section{Experimental design}

ENNG, purchased from Nacalai Tesque, Inc. (Kyoto), was dissolved in polyethylene glycol (PEG) at a $1.5 \%$ concentration just before use. $\mathrm{E}_{2}$ was obtained from Sigma ( $\mathrm{St}$ Louis, MO, USA), and pellets containing $0.32 \mathrm{mg}$ of $E_{2}$ and $31.68 \mathrm{mg}$ of cholesterol were made by the method described previously? ${ }^{7}$ At 10 weeks of age, mice in Groups 1 and 3 were given a single dose of PEG $(833 \mu \mathrm{l} / \mathrm{kg})$ into one of the uterine cavities using a $23 \mathrm{G}$ needle ( $45 \mathrm{~mm}$ in length) via the 


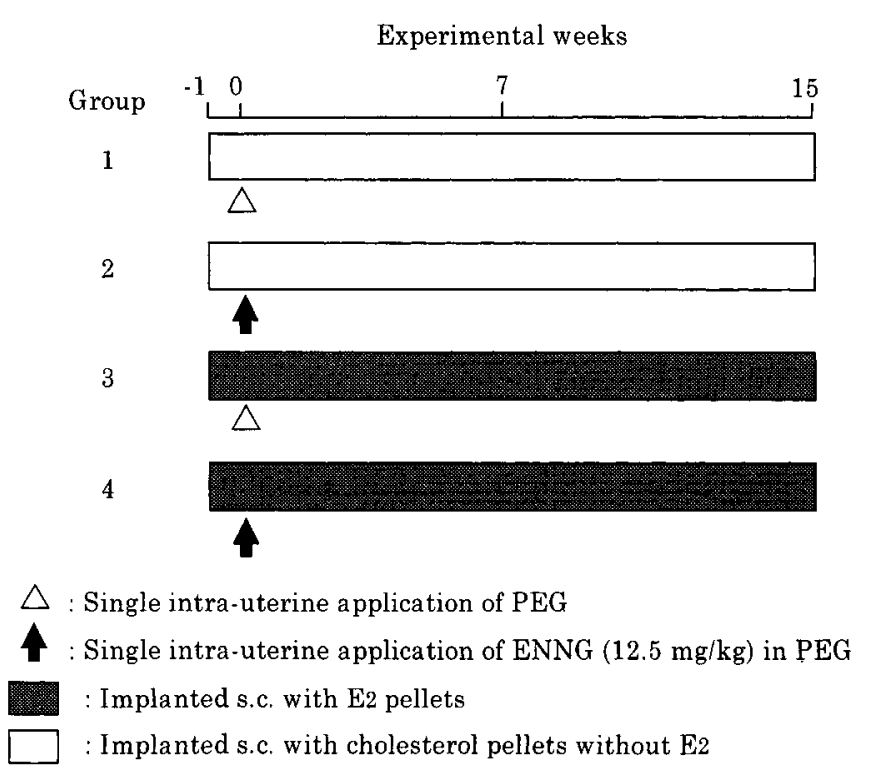

Fig. 1. Experimental design

vagina, and those in Groups 2 and 4 were given $12.5 \mathrm{mg} / \mathrm{kg}$ ENNG, dissolved in PEG, in the same manner. In Groups 3 and 4, mice were implanted s.c. with $\mathrm{E}_{2}$ pellets at 9 weeks of age ( 1 week before the ENNG treatment), and then the pellets were renewed after 8 weeks. $\mathrm{E}_{2}$ release, calculated from loss of pellet weight, was estimated to be $4.0 \pm 1.2 \mathrm{ng} \mathrm{E}_{2} /$ day. In Groups 1 and 2, animals were implanted s.c. with cholesterol pellets without $E_{2}$. The animals were observed daily for clinical signs and mortality, and water- and foodconsumption and body weights were recorded once a week during the experimental period.

The experiment was terminated at 15 weeks after the ENNG treatment, when all survivors were sacrificed.

\section{Histopathological examination}

All mice were autopsied and the uterus, ovaries, vagina, and other main organs (pituitary gland, mammary gland, adrenal gland, liver, spleen, kidney, heart, lung, and thymus) were removed, the first two being weighed, fixed in buffered $10 \%$ formalin and then routinely processed for histopathological examination. Sections were stained with hematoxylin and eosin. Each uterus was cut into 5-7 pieces, specimens from the uterine horns and the corpus uteri being sectioned transversally and the others longitudinally.

Uterine endometrial lesions were histologically classified into hyperplasia and adenocarcinoma categories, according to the diagnostic criteria reported previously ${ }^{5,7,9}$. Briefly, endometrial hyperplasias were divided into three degrees on the basis of size and atypia : slight $(+)$, moderate $(++)$, and severe $(+++)$, and endometrial adenocarcinomas were diagnosed on the basis of invasion of tumor cells into the muscularis.

\section{Hormone assays}

At the termination, 5 animals in each group were assessed for sex-steroid hormone levels. $E_{2}$ assays were performed with a double antibody technique using a specific sheep antiserum (GDN 244), as reported previously? ${ }^{7}$ Progesterone was radioimmunoassayed using a Progesterone kit 'Daiichi II' (Daiichi Radioisotope Lab. Ltd., Tokyo).

\section{Statistical analysis}

Data for incidences of endometrial proliferative lesions were analyzed using Fisher's exact probability test. Data for body and organ weights as well as hormone levels were assessed for statistical significance with the Student's $t$-test.

\section{Results}

General conditions, body weights, and uterus and ovary weights

No animals died during the experimental period, and no general clinical abnormalities were observed in any of the groups. Body weights did not significantly vary, while the relative uterus and ovary weights were significantly increased and decreased, respectively, by the $\mathrm{E}_{2}$ treatment $(p<0.001)$ (Table 1). The mean water intake in Groups 3 and 4 tended to be increased slightly, compared with that in Group 1 throughout the experimental period, although the mean food intake was the same in all groups (data not shown).

Incidences and histopathological findings of proliferative lesions in the uterns

The incidences of uterine endometrial hyperplasias and adenocarcinomas at terminal sacrifice are summarized in Table 2. Group 1 lesions were limited to slight and moderate endometrial hyperplasias. The incidences of adenocarcinomas in the $E_{2}$-treated Groups 3 and 4 were significant as compared to the lack in Group 1, the incidence in Group 4 being also significantly higher than that in Group 2 .

Histologically, slight/moderate endometrial hyperplasias were characterized by increased numbers of glands with slightly atypical cells in focal and/or diffuse areas of the endometrium, cystic dilatation of glands being obvious in some cases (Fig. 2). Severe hyperplasias were composed of marked proliferation of atypical glands, with crowding, stratification, and irregularity of cells, but without invasion

Table 1. Body and Relative Uterus and Ovary Weights

\begin{tabular}{lcccc}
\hline \multicolumn{1}{c}{ Group } & $\begin{array}{c}\text { No. of } \\
\text { animals }\end{array}$ & $\begin{array}{c}\text { Body weight } \\
(\mathrm{g})\end{array}$ & $\begin{array}{c}\text { Uterus } \\
(\mathrm{mg} / \mathrm{g} \text { body wt })\end{array}$ & $\begin{array}{c}\text { Ovary } \\
(\mathrm{mg} / \mathrm{g} \text { body wt })\end{array}$ \\
\hline 1 (Control) & 20 & $36.9 \pm 5.2^{\mathrm{a}}$ & $5.90 \pm 2.05$ & $0.83 \pm 0.25$ \\
$2($ ENNG) & 19 & $36.6 \pm 2.6$ & $5.69 \pm 1.87$ & $0.70 \pm 0.21$ \\
$3\left(\mathrm{E}_{2}\right)$ & 20 & $38.0 \pm 5.8$ & $10.69 \pm 2.74^{\mathrm{b}}$ & $0.40 \pm 0.13^{\mathrm{b}}$ \\
$4\left(\mathrm{ENNG}+\mathrm{E}_{2}\right)$ & 20 & $36.9 \pm 3.4$ & $8.85 \pm 2.08^{\mathrm{b}}$ & $0.40 \pm 0.11^{\mathrm{b}}$ \\
\hline
\end{tabular}

\footnotetext{
a Mean \pm SD

b Significant difference from Groups 1 and $2(p<0.001)$
} 
Table 2. Incidences of Endometrial Proliferative Lesions at the Terminal Sacrifice

\begin{tabular}{|c|c|c|c|c|c|c|}
\hline \multirow{2}{*}{ Group } & \multirow{2}{*}{$\begin{array}{l}\text { No. of } \\
\text { animals }\end{array}$} & \multirow{2}{*}{$\begin{array}{c}\text { All } \\
\text { proliferative } \\
\text { lesions }\end{array}$} & \multicolumn{3}{|c|}{ Hyperplasia } & \multirow{2}{*}{ Adenocarcinoma } \\
\hline & & & + & ++ & +++ & \\
\hline 1 (Control) & 20 & $11(55)^{\mathrm{a}}$ & $8(40)$ & $3(15)$ & $0(0)$ & $0(0)$ \\
\hline $2(\mathrm{ENNG})$ & 19 & $8(43)$ & $3(15)$ & $2(11)$ & $2(11)$ & $1(6)$ \\
\hline $3\left(\mathrm{E}_{2}\right)$ & 20 & $18(90)^{b}$ & $2(10)^{\mathrm{d}}$ & $8(40)^{e}$ & $3(15)$ & $5(25)^{\mathrm{d}}$ \\
\hline $4\left(\mathrm{ENNG}+\mathrm{E}_{2}\right)$ & 20 & $20(100)^{c}$ & $2(10)^{\mathrm{d}}$ & $3(15)$ & $4(20)$ & $11(55)^{c}$ \\
\hline
\end{tabular}

a Percentage in parentheses

b Significant difference from Groups 1 and $2(p<0.05)$

c Significant difference from Groups 1 and $2(p<0.001)$

d Significant difference from Group $1 \quad(p<0.05)$

e Significant difference from Group $2(p<0.05)$

into the muscularis (Fig. 3). Adenocarcinomas were composed of marked and irregular proliferations of atypical glands consisting of one or more layers of cuboidal of columnar cells with back-to-back arrangements and showing evidence of invasion into the muscularis (Fig. 4), although distant metastasis was not found in any cases.

As other uterine lesions, deciduomas were observed in 2 out of 19 animals (11\%) in Group 2, and a few cases of adenomyosis, diagnosed as focal or multifocal down-growth of glands into the myometrium with stromal components, were also found in Group 1 (15\%), Group 2 (16\%) and Group $3(5 \%)$.

In addition to the uterine lesions, ovarian atrophy was prominent in Groups 3 and 4 . Histologically, the numbers
Table 3. Serum Sex-steroid Hormone Concentrations and $\mathrm{E}_{2}$ : P Ratios

\begin{tabular}{lccc}
\hline \multicolumn{1}{c}{ Group } & $\begin{array}{c}\mathrm{E}_{2} \\
(\mathrm{pg} / \mathrm{ml})\end{array}$ & $\begin{array}{c}\text { Progesterone } \\
(\mathrm{ng} / \mathrm{ml})\end{array}$ & $\begin{array}{c}\mathrm{E}_{2}: \text { P ratio } \\
\left(\times 10^{-3}\right)\end{array}$ \\
\hline 1 (Control) & $13.3 \pm 2.6^{\mathrm{a}}$ & $8.60 \pm 0.75$ & $1.60 \pm 0.42$ \\
$2(\mathrm{ENNG})$ & $11.2 \pm 1.7$ & $8.85 \pm 1.49$ & $1.30 \pm 0.13$ \\
$3\left(\mathrm{E}_{2}\right)$ & $27.5 \pm 2.6^{\mathrm{b}}$ & $3.42 \pm 0.61^{\mathrm{b}}$ & $8.16 \pm 1.16^{\mathrm{b}}$ \\
$4\left(\mathrm{ENNG}+\mathrm{E}_{2}\right)$ & $30.3 \pm 3.1^{\mathrm{b}}$ & $3.85 \pm 1.65^{\mathrm{c}}$ & $8.92 \pm 3.19^{\mathrm{b}}$ \\
\hline
\end{tabular}

a Mean \pm SD

${ }^{b}$ Significant difference from Groups 1 and $2(p<0.001)$

c Significant difference from Groups $1(p<0.001)$ and $2(p<$ 0.01 )

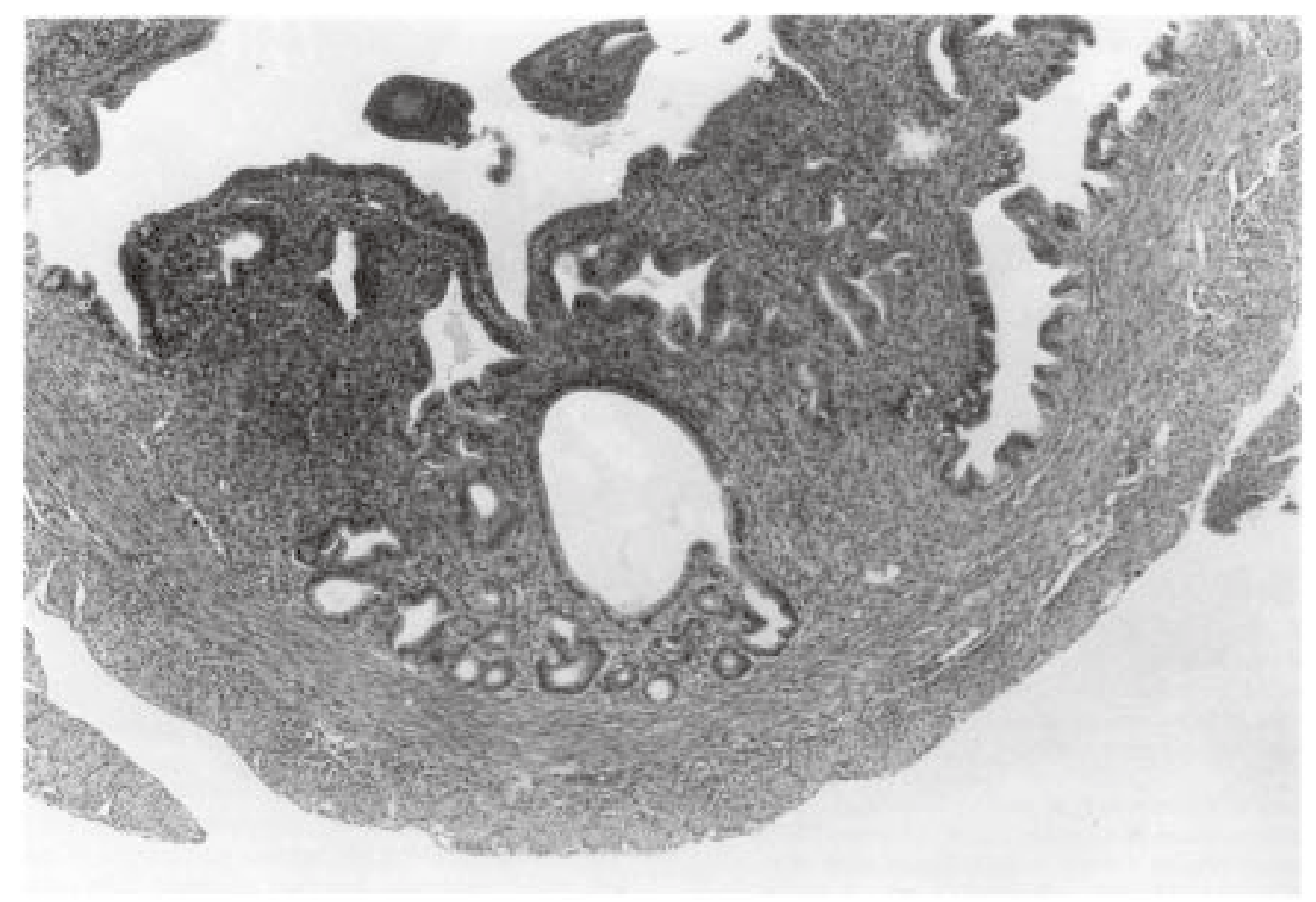

Fig. 2. Moderate endometrial hyperplasia in a group 2 mouse. Note focal proliferation of slightly atypical glands. H \& E. $\times 75$. 


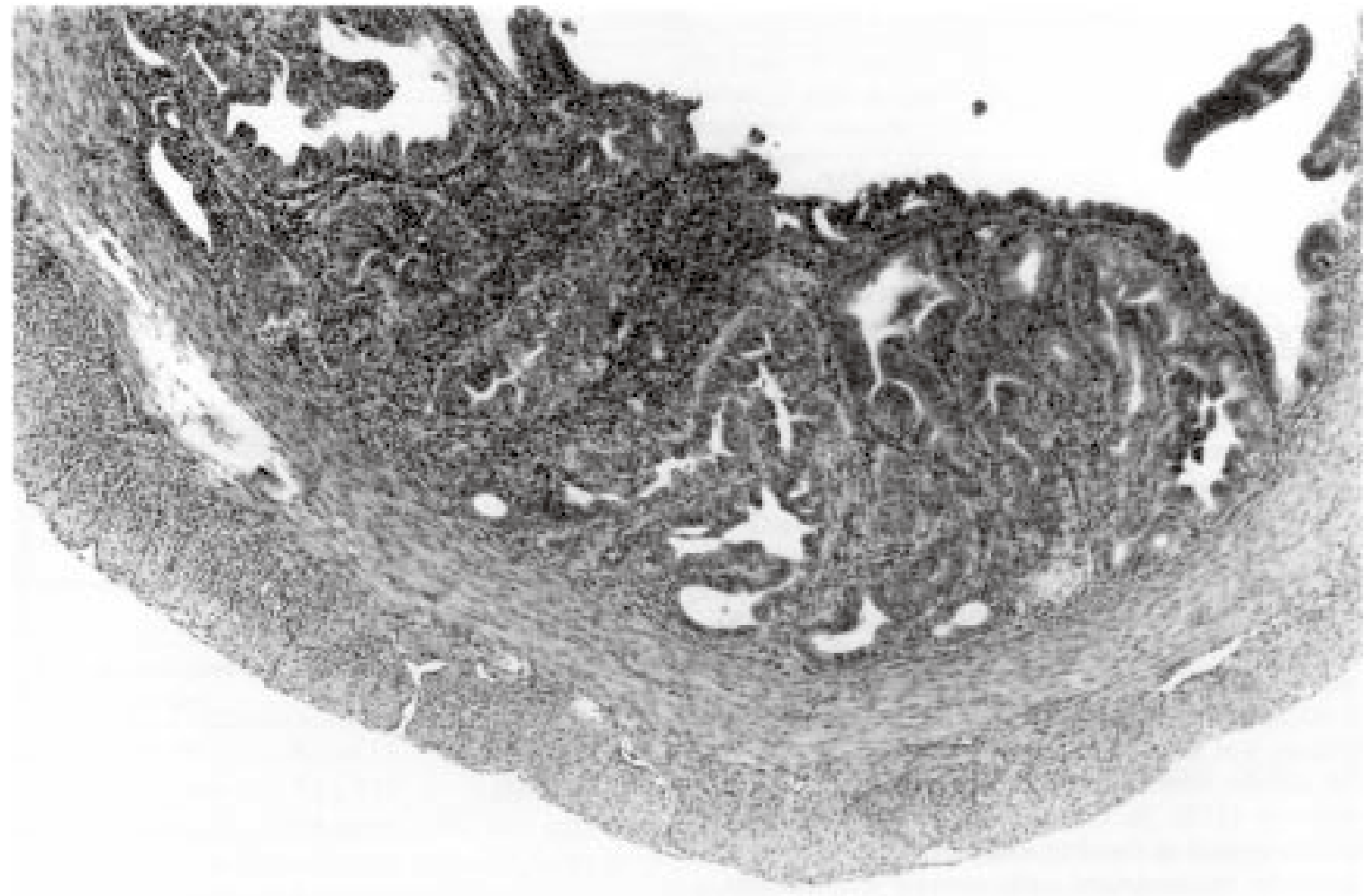

Fig. 3. Severe endometrial hyperplasia in a group 4 mouse. Diffuse and irregular proliferation of atypical glands is apparent without invasion into the muscularis. H\& E. $\times 75$.

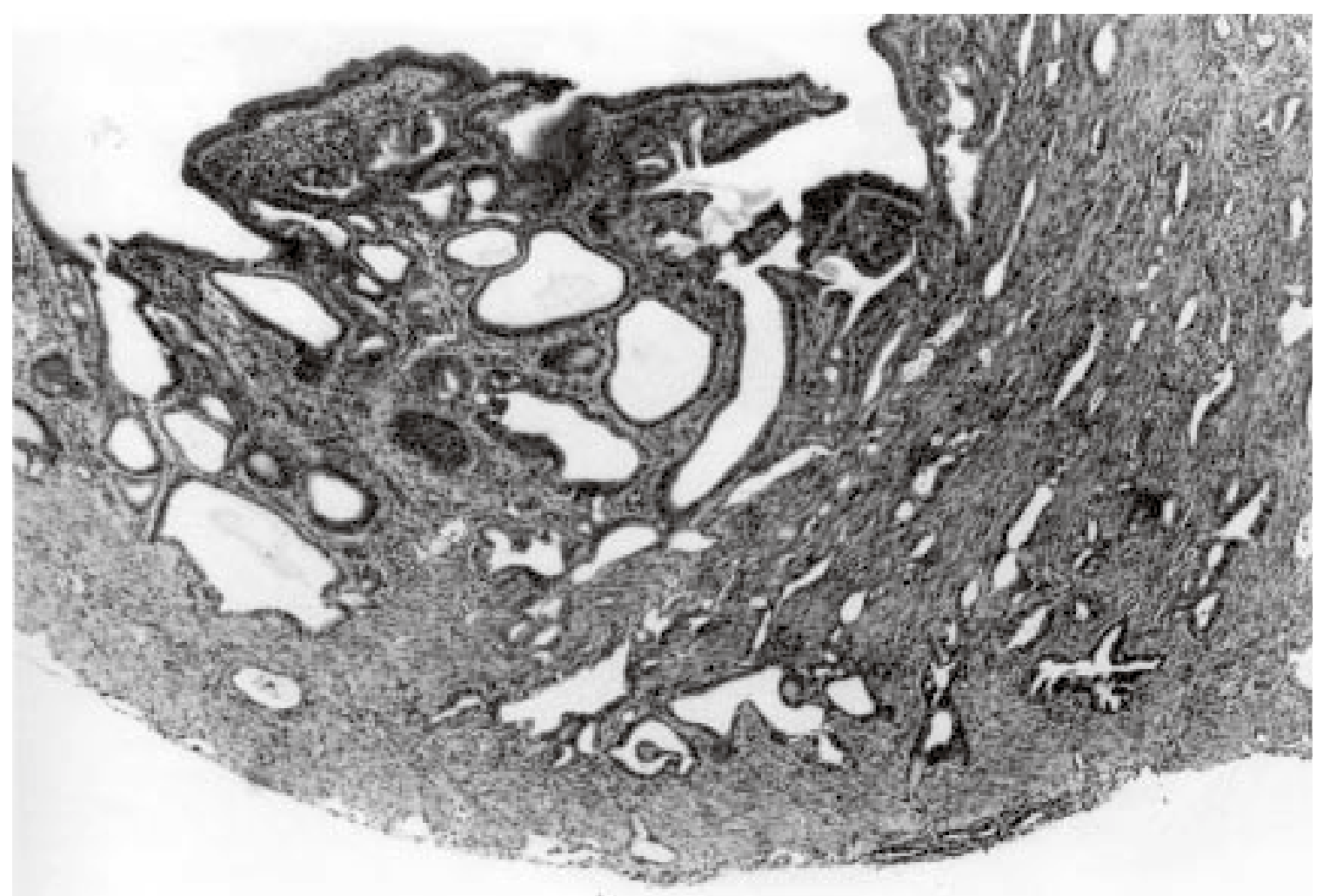

Fig. 4. Endometrial adenocarcinoma in a group 4 mouse. Proliferation and invasion of atypical glands into the muscularis are evident. H \& E. $\times 75$. 
of the follicle and corpus luteum, especially the latter, were decreased, and interstitial cells were increased in these groups. Cornification of the vaginal epithelium was also more frequently observed in the groups given $\mathrm{E}_{2}$ than Groups 1 and 2. No lung adenomas were found in any animals including ENNG-treated ones. In the other organs (pituitary, mammary, adrenal, liver, kidney, heart, spleen, and thymus), no ENNG and/or $\mathrm{E}_{2}$-treated lesions were evident.

\section{Serum sex-steroid hormone concentrations}

Table 3 summarizes data for serum $E_{2}$ and progesterone concentrations, and $E_{2}: P$ ratios in each group at the terminal sacrifice. There were no significant differences in serum $E_{2}$ and progesterone and $E_{2}: P$ ratios between Groups 1 and 2 , and also between Groups 3 and 4 , although the $E_{2}$ and progesterone values in the latter were significantly increased and decreased, respectively, compared with those in Groups 1 and 2 . Thus, the $E_{2}: P$ ratios in Groups 3 and 4 were very much higher than in the other two groups $(p<0.001)$.

\section{Discussion}

For animal models of human endometrial adenocarcinomas, $N$-nitroso compounds such as ENU, $N$-methyl$N^{\prime}$-nitro- $N$-nitrosoguanidine (MNNG), or ENNG have recently been employed, intra-uterine administration of these carcinogens causing cancers at relatively high incidence in rats and mice, as reviewed very recently ${ }^{3}$. In Donryu rats, Ando et $a l .^{5}$ reported high-yield induction of uterine endometrial adenocarcinomas by a single intra-uterine administration of ENNG via the vagina. Subsequently, Takahashi et al. ${ }^{7}$ described high and rapid induction of uterine endometrial adenocarcinomas in CD- 1 mice by ENU and $\mathrm{E}_{2}$. The latent period was shorter in mice than rats, although development of lung adenomas was also observed. In the present study, similar high yields of endometrial adenocarcinomas were specifically induced without toxicity, body weights not being affected, and only slight increase in water intake due to the diuretic effect of estrogen. The results thus indicate that ENNG is better than ENU for selective induction of endometrial carcinomas in mice.

In controls, slight endometrial hyperplasias were observed with relatively high incidence, in line with the previous report $^{7}$. Endometrial hyperplasias are common spontaneous lesions in aged mice of almost all strains including CD-1 mice, the incidences being increased age-dependently ${ }^{10}$. Relatively high yield of the lesion in the present study may depend on illumination-induced persistent estrous mice.

In humans, endometrial proliferative lesions are mainly classified into hyperplasias and adenocarcinomas, the former being divided into three categories such as cystic, adenomatous, and atypical. In the present study, the lesions were also classified into hyperplasia and adenocarcinoma categories, according to the diagnostic criteria reported previously in the case of rats $\mathbf{r}^{5,9}$

However, one disadvantage in mouse models is that the induced endometrial adenocarcinomas are better differentiated than those in rats ${ }^{5,9}$, and differential diagnosis from adenomyosis is difficult in some cases. This is very important, because adenomyosis occurs spontaneously in mice and can be induced by estrogen or prolactin administration $^{11}$. Differential diagnosis between adenocarcinoma and severe hyperplasia is also very important, but the diagnostic criteria are not yet established in rodents including mouse.
In humans also, differential diagnosis from atypical hyperplasias is a very important problem. Thus, further studies are needed to harmonize the technical terms of endometrial lesions between humans and animals and also to establish the diagnostic criteria of the lesions in rodents including mouse.

It has been reported that the mouse is susceptible to uterine carcinogenicity of estrogen alone ${ }^{3,4,10}$, this being confirmed here for CD-1 mice. In addition, the incidence of adenocarcinomas in Group 4 was higher than that in Group 3 , although the difference was not significant. Thus, the results indicate that $E_{2}$ acts as both a promoter and a complete carcinogen in the endometrium.

In rodents, a hormonal imbalance leading to an increased $E_{2}$ : $\mathbf{P}$ ratio has been shown to be very important for development of endometrial cancers ${ }^{3}$. In the present study, significant increase of $\mathrm{E}_{2}$ and decrease of progesterone concentrations were detected in $\mathrm{E}_{2}$-treated mice. These are expected from reduction in gonadotropin-release into the blood stream from the anterior pituitary gland through negative feedback, as reflected in the uterine and ovary weights. Histological changes of the ovary and vagina in Group 3 and 4 reflect also the result. The increased $E_{2}: P$ ratio in the present study was also considerd to be presumably important for the development of endometrial adenocarcinomas, in line with the previous report ${ }^{7}$.

Recently, possible adverse consequences arising from the release of man-made substances with estrogenic properties into the environment, so-called endocrine disruptors, have become an important social concern. Carcinogenic effects are suspected in some cases, so that evaluation of the risk of these chemicals to the female genital organs including the uterus is of high priority. The experimental model using ENNG and $E_{2}$ described in the present study provides a good method for two-stage uterine carcinogenicity studies in mice, with rapid induction of cancers, so that it should find application for risk assessment purpose.

Acknowledgements : This study was supported by Grantsin-Aid from the Ministry of Health and Welfare of Japan.

\section{References}

1. Zeil HK. Estrogen's role in endometrial cancer. Obstet Gynecol 1982; 60: 509-515.

2. Fox H. Endometrial carcinogenesis and its relation to oestrogens. Pathol Res Pract 1984; $179: 13-19$

3. Maekawa A, Takahashi M, Ando J, and Yoshida M. Uterine carcinogenesis by chemicals/hormones in rodents. J Toxicol Pathol 1999; 12: 1-11.

4. Newbold RR, Bullock BC, and McLachlan JA. Uterine adenocarcinoma in mice following developmental treatment with estrogens: A model for hormonal carcinogenesis. Cancer Res 1990; 50:7677-7681.

5. Ando-Lu J, Takahashi M, Imai S, Ishihara R, Kitamura T, Iijima T, Takano S, Nishiyama K, Suzuki K, and Maekawa A. High-yield induction of uterine endometrial adenocarcinomas in Donryu rats by a single intra-uterine administration of $N$ ethyl- $N^{\prime}$-nitro- $N$-nitrosoguanidine via the vagina. Jpn J Cancer Res 1994; 85: 789-793.

6. Niwa K, Tanaka T, Mori H, Yokoyama Y, Furui T, Mori H, and Tanaka T. Rapid induction of endometrial carcinoma in ICR mice treated with $N$-methyl- $N$-nitrosourea and $17 \beta-$ estradiol. Jpn J Cancer Res 1991; 82 : 1391-1396.

7. Takahashi M, Iijima T, Suzuki K, Ando-Lu J, Yoshida M, Kitamura T, Nishiyama K, Miyajima K, and Maekawa A. 
Rapid and high yield induction of endometrial adenocarcinomas in $\mathrm{CD}-1$ mice by a single intrauterine administration of $N$-ethyl-N-nitrosourea combined with chronic $17 \beta-$ estradiol treatment. Cancer Lett $1996 ; 104: 7-12$.

8. IARC (International Agency for Research on Cancer). Some $N$-nitroso compounds. In: IARC Monographs on the evaluation of carcinogenic risk of chemicals to man (vol. 17), Lyon: IARC, 227-255, 1978.

9. Nagaoka T, Takeuchi M, Onodera H, Matsushima Y, AndoLu J, and Maekawa A. Sequential observation of spontaneous endometrial adenocarcinoma development in Donryu rats.
Toxicol Pathol $1994 ; 22: 261-269$

10. Maekawa $\mathrm{A}$, and Maita $\mathrm{K}$. Changes in the uterus and vagina. In : Pathobiology of the Aging Mouse (Vol. 1), U Mohr, DL Dungworth, CC Capen, WW Carlton, JP Sundberg and JP Ward (eds), Washington DC : ILSI Press, 469-480, 1996.

11. Maekawa A, and Yoshida A. Susceptibility of the female genital system to toxic substances. In : Pathobiology of the Aging Mouse (Vol. 1), U Mohr, DL Dungworth, CC Capen, WW Carlton, JP Sundberg and JP Ward (eds), Washington DC : ILSI Press, 481-493, 1996. 